\title{
Correction to: Criminal Behavior and School Discipline in Juvenile Justice-Involved Youth with Autism
}

\author{
Alexandra M. Slaughter ${ }^{1}$ - Sascha Hein ${ }^{1}\left[\right.$ - Judy H. Hong ${ }^{2}$ - Sarah S. Mire ${ }^{1}$ Elena L. Grigorenko ${ }^{2,3,4}$ \\ Published online: 4 April 2020 \\ ○) Springer Science+Business Media, LLC, part of Springer Nature 2020

\section{Correction to:} \\ Journal of Autism and Developmental Disorders \\ Publisher's Note Springer Nature remains neutral with regard to \\ jurisdictional claims in published maps and institutional affiliations.
} (2019) 49:2268-2280

https://doi.org/10.1007/s10803-019-03883-8

Unfortunately, the original version of the article contained formatting errors within Tables 1, 2, and 3. The correct tables are shown below.

The original article can be found online at https://doi.org/10.1007/ s10803-019-03883-8.

Elena L. Grigorenko

elena.grigorenko@times.uh.edu

1 Department of Psychological, Health, and Learning

Sciences, University of Houston, Houston, TX, USA

2 Department of Psychology, University of Houston, Houston, TX, USA

3 Texas Institute for Measurement, Evaluation, and Statistics, University of Houston, Houston, TX 77204-6022, USA

4 Laboratory of Translational Sciences of Human Development, Saint Petersburg State University, Saint Petersburg, Russia 
Table 1 Number and overall percentage of JJY with autism, JJY without SEN, JJY with SEN, and JJY with LD (n $=572)$ who committed at least one offense prior to (2006-2010) and in the main study year (2010-2011 school year)

\begin{tabular}{|c|c|c|c|c|c|c|c|c|c|c|c|}
\hline \multirow[t]{2}{*}{ Offense } & \multicolumn{2}{|c|}{ JJY with autism } & \multicolumn{2}{|c|}{ JJY without SEN } & \multicolumn{2}{|c|}{ JJY with SEN } & \multicolumn{2}{|c|}{ JJY with LD } & \multirow[t]{2}{*}{$\chi^{2}$} & \multirow[t]{2}{*}{ FDR-adj. $p$} & \multirow[t]{2}{*}{$V$} \\
\hline & Number & $\%$ & Number & $\%$ & Number & $\%$ & Number & $\%$ & & & \\
\hline \multicolumn{12}{|c|}{ Main study year (2010-2011) } \\
\hline Crimes against persons & VS & 2.8 & VS & 2.8 & 12 & 8.4 & VS & 3.5 & 7.49 & 0.13 & .11 \\
\hline Status offenses & 10 & 7.0 & VS & 3.5 & 12 & 8.4 & 13 & 9.1 & 4.09 & 0.31 & .09 \\
\hline Property violations & VS & 0.7 & 14 & 9.8 & 7 & 4.9 & 15 & 10.5 & 14.88 & 0.02 & .16 \\
\hline Drug law violations & VS & 0.7 & 6 & 4.2 & VS & 1.4 & VS & 2.8 & 4.83 & 0.28 & .09 \\
\hline Public order & 10 & 7.0 & 15 & 10.5 & 23 & 16.1 & 13 & 9.1 & 6.81 & 0.16 & .11 \\
\hline \multicolumn{12}{|l|}{ Prior (2006-2010) } \\
\hline Crimes against persons & 33 & 23.1 & 22 & 15.4 & 34 & 23.8 & 23 & 16.1 & 5.42 & 0.22 & .10 \\
\hline Status offenses & 27 & 18.9 & 16 & 11.2 & 32 & 22.4 & 24 & 16.8 & 6.58 & 0.16 & .11 \\
\hline Property offenses & 12 & 8.4 & 34 & 23.8 & 32 & 22.4 & 28 & 19.6 & 13.85 & 0.02 & .16 \\
\hline Drug law violations & 2 & 1.4 & 10 & 7.0 & 10 & 7.0 & 6 & 4.2 & 6.61 & 0.16 & .11 \\
\hline Public order & 42 & 29.4 & 48 & 33.6 & 47 & 32.9 & 41 & 28.7 & 1.21 & 0.77 & .75 \\
\hline
\end{tabular}

Prior incidents include 2006-2010 school years. VS = value suppressed for variables with five or less individuals. FDR-adj. $p=p$-value adjusted for false discovery rate (Benjamini and Hochberg 1995). Bolded Chi-square indicates statistical significance $p<.05$

SEN special educational needs 
Table 2 Number and overall percentage of JJY with autism, JJY without SEN, JJY with SEN, and JJY with LD $(n=572)$ with at least one school discipline incident prior to (2006-2010) and in main study year (2010-2011)

\begin{tabular}{|c|c|c|c|c|c|c|c|c|c|c|c|}
\hline \multirow[t]{2}{*}{ Discipline } & \multicolumn{2}{|l|}{$\begin{array}{l}\text { JJY with } \\
\text { autism }\end{array}$} & \multicolumn{2}{|c|}{$\begin{array}{l}\text { JJY without } \\
\text { SEN }\end{array}$} & \multicolumn{2}{|c|}{ JJY with SEN } & \multicolumn{2}{|c|}{ JJY with LD } & \multirow[t]{2}{*}{$\chi^{2}$} & \multirow[t]{2}{*}{ FDR-adj. $p$} & \multirow[t]{2}{*}{$V$} \\
\hline & Number & $\%$ & Number & $\%$ & Number & $\%$ & Number & $\%$ & & & \\
\hline \multicolumn{12}{|c|}{ Main study year (2010-2011) } \\
\hline Violent & VS & 1.4 & VS & 0.7 & VS & 0.7 & VS & 0.0 & 2.79 & 0.46 & .06 \\
\hline Sexual & VS & 0.0 & VS & 0.7 & 6 & 4.2 & VS & 2.1 & 9.92 & 0.06 & .12 \\
\hline Threatening & 7 & 4.9 & 8 & 5.6 & 17 & 11.9 & 7 & 4.9 & 7.79 & 0.12 & .12 \\
\hline Theft & VS & 0.7 & VS & 0.0 & VS & 1.4 & VS & 1.4 & 3.33 & 0.41 & .06 \\
\hline Confrontation & VS & 2.8 & 12 & 8.4 & 14 & 9.8 & 10 & 7.0 & 6.02 & 0.18 & .10 \\
\hline Fighting & 7 & 4.9 & 12 & 8.4 & 21 & 14.7 & 15 & 10.5 & 8.27 & 0.11 & .12 \\
\hline Property & VS & 0.7 & VS & 1.4 & VS & 0.7 & VS & 3.5 & 4.35 & 0.30 & .09 \\
\hline Weapons & VS & 0.7 & VS & 1.4 & VS & 2.1 & 6 & 4.2 & 4.60 & 0.29 & .09 \\
\hline Drug incidents & VS & 1.4 & VS & 2.8 & VS & 2.1 & 11 & 7.7 & 10.36 & 0.06 & .14 \\
\hline Policy & 17 & 11.9 & 33 & 23.1 & 46 & 32.2 & 48 & 33.6 & 22.79 & 0.00 & .20 \\
\hline OSS & 13 & 9.1 & 25 & 17.5 & 44 & 30.8 & 39 & 27.3 & 24.77 & 0.00 & .21 \\
\hline ISS & 22 & 15.4 & 35 & 24.5 & 43 & 30.1 & 48 & 33.6 & 14.07 & 0.02 & .16 \\
\hline EXP & VS & 0.7 & VS & 2.1 & VS & 0.0 & VS & 0.7 & 4.39 & 0.30 & .08 \\
\hline \multicolumn{12}{|c|}{ Prior (2006-2010) } \\
\hline Violent & VS & 2.1 & VS & 2.1 & 8 & 5.6 & VS & 0.0 & 11.61 & 0.04 & .13 \\
\hline Sexual & VS & 2.8 & 7 & 4.9 & 7 & 4.9 & 6 & 4.2 & 1.04 & 0.79 & .04 \\
\hline Threatening & 25 & 17.5 & 23 & 16.1 & 43 & 30.1 & 30 & 21.0 & 10.18 & 0.06 & .13 \\
\hline Theft & 10 & 7.0 & 7 & 4.9 & 11 & 7.7 & 12 & 8.4 & 1.51 & 0.72 & .05 \\
\hline Confrontation & 29 & 20.3 & 26 & 18.2 & 50 & 35.0 & 36 & 25.2 & 12.90 & 0.03 & .15 \\
\hline Fighting & 38 & 26.6 & 29 & 20.3 & 47 & 32.9 & 42 & 29.4 & 6.14 & 0.18 & .10 \\
\hline Property & VS & 2.1 & 10 & 7.0 & 6 & 4.2 & 8 & 5.6 & 4.16 & 0.31 & .09 \\
\hline Weapons & 9 & 6.3 & 10 & 7.0 & 12 & 8.4 & VS & 3.5 & 3.08 & 0.43 & .07 \\
\hline Drug incidents & VS & 2.1 & 12 & 8.4 & 12 & 8.4 & 7 & 4.9 & 7.13 & 0.14 & .11 \\
\hline Policy & 55 & 38.5 & 72 & 50.3 & 84 & 58.7 & 71 & 49.7 & 11.89 & 0.03 & .14 \\
\hline OSS & 67 & 46.9 & 61 & 42.7 & 83 & 58.0 & 77 & 53.8 & 8.17 & 0.11 & .12 \\
\hline ISS & 49 & 34.3 & 73 & 51.0 & 76 & 53.1 & 63 & 44.1 & 12.54 & 0.03 & .15 \\
\hline EXP & VS & 0.7 & VS & 3.5 & VS & 2.8 & VS & 2.8 & 3.26 & 0.41 & .07 \\
\hline
\end{tabular}

Prior incidents include 2006-2010 school years. FDR-adj. $p=p$ value adjusted for false discovery rate (Benjamini and Hochberg 1995). Bolded Chi-square indicates statistical significance, FDR-adj. $p<.05$

OSS out-of-school suspension, ISS in-school suspension, EXP expulsion 
Table 3 Logistic regression models predicting recidivism $(\mathrm{n}=308)$

\begin{tabular}{|c|c|c|c|c|c|c|}
\hline \multirow[t]{2}{*}{ Variable } & \multicolumn{2}{|c|}{ Model 1} & \multicolumn{2}{|l|}{ Model 2} & \multicolumn{2}{|l|}{ Model 3} \\
\hline & $\overline{\mathrm{B}}$ & $\overline{\mathrm{OR}}$ & $\overline{\mathrm{B}}$ & OR & $\bar{B}$ & OR \\
\hline No special education & 0.12 & 1.13 & 0.12 & 1.13 & 0.36 & 1.45 \\
\hline Special education & $0.72 *$ & 2.06 & 0.58 & 1.79 & 0.54 & 1.72 \\
\hline Learning disability & 0.46 & 1.58 & 0.40 & 1.48 & 0.11 & 1.12 \\
\hline Out-of-school-suspension & & & 0.04 & 1.04 & -0.34 & 0.71 \\
\hline In-school-suspension & & & -0.26 & 0.77 & 0.20 & 1.22 \\
\hline Threatening & & & 0.03 & 1.03 & 0.06 & 1.06 \\
\hline Confrontation & & & 0.09 & 1.10 & 0.10 & 1.10 \\
\hline Fighting & & & $0.72 *$ & 2.06 & $0.70^{*}$ & 2.01 \\
\hline Policy & & & 0.44 & 1.55 & 0.33 & 1.39 \\
\hline OSS*no special education & & & & & 0.73 & 2.06 \\
\hline ISS*no special education & & & & & -1.14 & 0.32 \\
\hline OSS*special education & & & & & 0.89 & 2.42 \\
\hline ISS*special education & & & & & -0.94 & 0.39 \\
\hline OSS*learning disability & & & & & 0.20 & 1.23 \\
\hline ISS*learning disability & & & & & 0.22 & 1.24 \\
\hline \multicolumn{7}{|l|}{ Fit statistics } \\
\hline Chi-square & \multicolumn{2}{|r|}{5.81} & \multicolumn{2}{|c|}{18.52} & \multicolumn{2}{|c|}{23.74} \\
\hline Nagelkerke $R^{2}$ & \multicolumn{2}{|r|}{.025} & \multicolumn{2}{|c|}{.080} & \multicolumn{2}{|c|}{.101} \\
\hline
\end{tabular}

The special education variables was coded $0=$ JJY without SEN, JJY with SEN, JJY with LD and $1=$ JJY with autism. JJY with autism was the reference group. Prior school discipline variables (2006-2010) were coded $0=$ no school discipline incidents and $1=$ one or more school discipline incidents in each respective category (i.e., OSS, ISS, threatening, confrontation, fighting, property, drugs, and policy violations)

$O R$ odds ratio

${ }^{*} p<.05$ 